\title{
Recent Trends in Euthanasia and Other End-of-Life Practices in Belgium
}

TO THE EDITOR: In Belgium, where euthanasia was legalized in 2002, large-scale repeat surveys have monitored the evolution of medical end-oflife practices since 1998, with subsequent surveys conducted in 2001 and $2007^{1,2}$ and the latest in 2013.

As was done in previous surveys, ${ }^{2}$ we sent questionnaires to 6188 physicians certifying death certificates from the first half of 2013 in Flanders, the Dutch-speaking half of Belgium, with approximately 6 million inhabitants and 58,000 deaths annually (see the Supplementary Appendix, available with the full text of this letter at NEJM.org). The response rate was $60.6 \%$. The response sample was weighted to be representative of all the deaths that occurred in the first half of 2013.

After a large increase between 2001 and 2007, the total percentage of deaths preceded by one or more possibly life-shortening end-of-life practices remained stable at $47.8 \%$ in 2013 (Table 1). The intensified alleviation of pain and other symptoms with the use of drugs, with possible shortening of life taken into account $(24.2 \%$ of deaths), and the withholding or withdrawing of life-prolonging treatment $(17.2 \%)$ remained the most prevalent end-of-life practices.

The rate of euthanasia increased significantly between 2007 and 2013, from 1.9 to $4.6 \%$ of deaths. The overall increase relates to increases in both the number of requests (from 3.5 to $6.0 \%$ of deaths) and the proportion of requests granted (from 56.3 to $76.8 \%$ of requests made).

After a decrease from 3.2\% in 1998 to $1.8 \%$ in 2007 , the rate of hastening death without an explicit request from the patient remained stable at $1.7 \%$ in 2013. After an increase from $8.2 \%$ in 2001 to $14.5 \%$ in 2007 , the rate of use of continuous deep sedation until death decreased to $12.0 \%$ in 2013.

As compared with practices in 2007 , decision making in euthanasia and physician-assisted suicide in 2013 more often included an oral and written request from the patient and consultation with another physician, both of which are requirements of the euthanasia law ${ }^{3}$ (Table S1 in the Supplementary Appendix). Palliative care services were involved in $73.7 \%$ of cases in 2013. These results suggest a stricter assessment of legal eligibility criteria in 2013 than in 2007. Decision making in other end-of-life practices also increasingly included patient and family input (data not shown).

We found an increased demand for euthanasia in Belgium between 2007 and 2013, as well as growing willingness among physicians to 


\begin{tabular}{|c|c|c|c|c|c|}
\hline \multirow[t]{2}{*}{ Variable } & \multicolumn{4}{|c|}{ Survey Year } & \multirow[t]{2}{*}{ P Value } \\
\hline & 1998 & 2001 & 2007 & 2013 & \\
\hline Total deaths annually - no. & 56,354 & 55,793 & 54,881 & $61,621 \Varangle$ & - \\
\hline Deaths in survey sample - no. & 3999 & 5005 & 6202 & 6188 & - \\
\hline Response rate - \% of physicians surveyed & 48.1 & 58.9 & 58.4 & 60.6 & - \\
\hline Studied cases - no. & 1925 & 2950 & 3623 & 3751 & - \\
\hline $\begin{array}{l}\text { Death preceded by at least one end-of-life prac- } \\
\text { tice - } \%(95 \% \mathrm{Cl})\end{array}$ & $39.3(37.0-41.6)$ & $38.4(36.5-40.3)$ & $47.8(45.9-49.8)$ & $47.8(46.1-49.5)$ & $>0.99$ \\
\hline $\begin{array}{l}\text { Intensified alleviation of pain and other } \\
\text { symptoms } \rrbracket\end{array}$ & $18.4(16.6-20.4)$ & $22.0(20.5-23.6)$ & $26.7(25.1-28.4)$ & $24.2(22.9-25.7)$ & 0.02 \\
\hline $\begin{array}{l}\text { Withholding or withdrawing of life-prolong- } \\
\text { ing treatment }\end{array}$ & $16.4(14.7-18.3)$ & $14.6(13.2-16.0)$ & $17.4(15.9-19.0)$ & $17.2(15.9-18.6)$ & 0.85 \\
\hline Physician-assisted death & $4.4(3.5-5.5)$ & $1.8(1.4-2.4)$ & $3.8(3.2-4.5)$ & $6.3(5.6-7.1)$ & $<0.001$ \\
\hline Euthanasia & $1.1(0.7-1.7)$ & $0.3(0.2-0.5)$ & $1.9(1.6-2.3)$ & $4.6(4.0-5.2)$ & $<0.001$ \\
\hline Assisted suicide & $0.12(0.04-0.36)$ & $0.01(0.00-0.10)$ & $0.07(0.02-0.19)$ & $0.05(0.02-0.13)$ & 0.97 \\
\hline $\begin{array}{l}\text { \% of deaths with request for eutha- } \\
\text { nasia or assisted suicide }\end{array}$ & $2.1(1.6-2.9)$ & $-\|$ & $3.5(3.0-4.1)$ & $6.0(5.3-6.7)$ & $<0.001$ \\
\hline $\begin{array}{l}\% \text { of requests for euthanasia or as- } \\
\text { sisted suicide granted }\end{array}$ & $57.4(41.0-72.4)$ & NC & $56.3(48.2-64.0)$ & $76.8(71.2-81.6)$ & $<0.001$ \\
\hline $\begin{array}{l}\text { Hastening of death without explicit re- } \\
\text { quest from patient }\end{array}$ & $3.2(2.4-4.1)$ & $1.5(1.1-2.0)$ & $1.8(1.3-2.4)$ & $1.7(1.3-2.2)$ & 0.84 \\
\hline $\begin{array}{l}\text { Continuous deep sedation until death - } \\
\qquad \%(95 \% \mathrm{Cl})\end{array}$ & $-\|$ & $8.2(7.1-9.4)$ & 14.5 (13.1-15.9) & $12.0(10.9-13.2)$ & 0.002 \\
\hline $\begin{array}{l}\text { Patient decided to stop eating and drinking - } \\
\qquad \%(95 \% \mathrm{Cl})\end{array}$ & $-\|$ & $-\|$ & $-\|$ & $0.5(0.3-0.7)$ & NC \\
\hline
\end{tabular}

* Data regarding these practices are weighted percentages with $95 \%$ confidence intervals $(\mathrm{Cl})$. Analyses were performed with the use of the complex samples function of SPSS software, version 22.0 (IBM). NC denotes could not be calculated.

$\dagger$ Two-sided P values are for the comparison of 2007 data with 2013 data and were calculated with the use of Pearson's chi-square test. Data are preliminary and unconfirmed, as reported by Statistics Belgium.

$\int$ Intensified alleviation of pain and other symptoms was performed with the use of drugs, with possible shortening of life taken into account.

I Physician-assisted death was defined as the administration of drugs with the explicit intention of shortening life. Euthanasia was defined as physician-assisted death at the explicit request of the patient.

|| The survey question was not asked in the respective year.

meet those requests, mostly after the involvement of palliative care services. This finding indicates that, after 11 years of experience, euthanasia is increasingly considered as a valid option at the end of life in Belgium. For the first time, the rate of euthanasia in the Flanders area of Belgium is significantly higher than that in the Netherlands $\left(2.8 \%\right.$ in 2010). ${ }^{4}$

Kenneth Chambaere, Ph.D.

Vrije Universiteit Brussel

Brussels, Belgium

kenneth.chambaere@vub.ac.be
Robert Vander Stichele, M.D., Ph.D.

Ghent University Hospital

Ghent, Belgium

Freddy Mortier, Ph.D.

Ghent University

Ghent, Belgium

Joachim Cohen, Ph.D.

Luc Deliens, Ph.D.

Vrije Universiteit Brussel

Brussels, Belgium

Drs. Cohen and Deliens contributed equally to this letter.

Supported by a grant (100036) from Agentschap voor Innovatie door Wetenschap en Technologie, the Flemish government agency for Innovation by Science and Technology. 
Disclosure forms provided by the authors are available with the full text of this letter at NEJM.org.

This letter was published on March 17, 2015, at NEJM.org.

1. Bilsen J, Cohen J, Chambaere K, et al. Medical end-of-life practices under the euthanasia law in Belgium. $\mathrm{N}$ Engl J Med 2009;361:1119-21.

2. Chambaere K, Bilsen J, Cohen J, Onwuteaka-Philipsen BD, Mortier F, Deliens L. Trends in medical end-of-life decision making in Flanders, Belgium 1998-2001-2007. Med Decis Making 2011;31:500-10.
3. Belgian Official Collection of the Laws. The Belgian Act on Euthanasia of May 28th 2002. Ethical Perspect 2002;9: 182-8.

4. Onwuteaka-Philipsen BD, Brinkman-Stoppelenburg A, Penning C, de Jong-Krul GJ, van Delden JJ, van der Heide A. Trends in end-of-life practices before and after the enactment of the euthanasia law in the Netherlands from 1990 to 2010: a repeated cross-sectional survey. Lancet 2012;380:908-15.

DOI: 10.1056/NEJMc1414527 


\section{Supplementary Appendix}

This appendix has been provided by the authors to give readers additional information about their work.

Supplement to: Chambaere K, Vander Stichele R, Mortier F, Cohen J, Deliens L. Recent trends in euthanasia and other end-of-life practices in Belgium. N Engl J Med. DOI: 10.1056/NEJMc1414527 
Table of contents

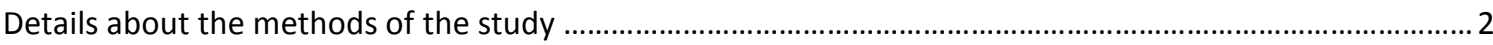

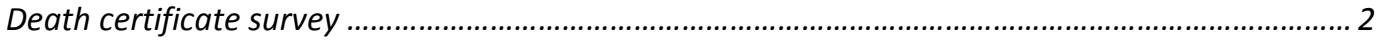

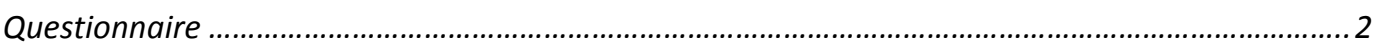

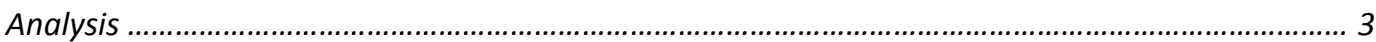

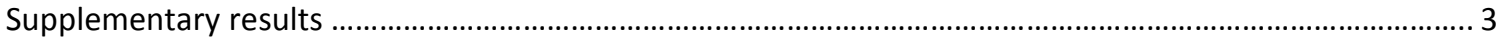

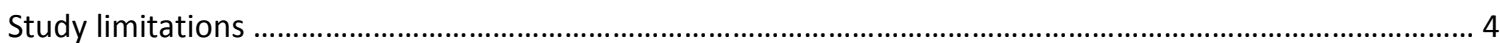

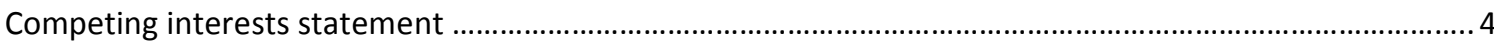




\section{Details about the methods of the study}

\section{Death certificate survey}

We conducted a population-based death certificate survey identical to surveys in 1998, 2001 and 2007, based on a large and representative sample of deaths in Flanders, the semi-autonomous northern half of Belgium with approximately six million inhabitants and 58,000 deaths annually. A stratified random sample of deaths in 2013 was drawn weekly at the Flemish Agency for Care and Health, the central administration authority for processing death certificates. All deaths from January $1^{\text {st }}$ until June $30^{\text {th }} 2013$ of Belgian residents aged one year or older were assigned to one of three strata, based on underlying cause of death as indicated on the death certificate and the estimated corresponding likelihood of an end-of-life decision. Sampling fractions for each stratum increased with this likelihood. In the first stratum, all deaths for which euthanasia was mentioned on the death certificate were sampled. In the second stratum, one third of all cancer deaths were sampled. In the third stratum, one in six deaths resulting from any other cause was sampled. This resulted in a sample of 6,871 deaths, about $21 \%$ of all deaths in the studied period.

Within two months of the death certifying physicians received a four-page questionnaire with an introductory letter containing patient identifiers. Physicians were requested to complete the questionnaire by consulting the patient's medical file. If the certifying physician was not the treating physician, the questionnaire was passed on to the treating physician. One physician could receive participation requests for up to five decedents, with at most three reminders per death case. To guarantee absolute anonymity for participating physicians, a lawyer served as an intermediary between responding physicians, researchers and the Flemish Agency for Care and Health, ensuring that completed questionnaires could never be linked to a particular patient or physician. Returning the questionnaire was regarded as implicit consent of the physician to participate in the study. After data collection a one-page questionnaire was mailed to all non-responding physicians inquiring about reasons for not participating. The mailing and anonymity procedures were approved by the Ethical Review Board of the University Hospital of the Vrije Universiteit Brussel, the Belgian National Disciplinary Board of Physicians, and the Belgian Privacy Commission.

\section{Questionnaire}

The repeatedly validated questionnaire on end-of-life decision-making first asked whether death had been sudden and unexpected. If answered negatively - and an end-of-life decision could thus not be precluded physicians were asked whether they had: (1) withheld or withdrawn life-prolonging medical treatment taking into account or explicitly intending hastening the patient's death; (2) intensified the alleviation of pain and/or other symptoms with drugs taking into account or co-intending possible hastening of death; and (3) administered, supplied or prescribed drugs with the explicit intention of hastening death. If in the latter case the drugs had been administered at the patient's explicit request, the act was classified as euthanasia or assisted suicide depending on whether the patient self-administered the drugs. If drugs were used with the same explicit intention to hasten death but without the patient's explicit request, the act was classified as hastening death without explicit patient request. This can include cases where a patient request was not judged as explicit by the physician, where the request came from the family or where the physician acted out of compassion.

If more than one end-of-life decision was made, the act that involved the most explicit life-shortening intention was used to classify the case. When two decisions with similar life-shortening intention were made, administering drugs prevailed over withholding or withdrawing treatment. Details about the decision-making process, the types of drugs used and the estimated degree of life shortening according to the physician were requested for the most important end-of-life decision. Further in the questionnaire, physicians were asked whether the patient had been deeply and continuously sedated until death, whether palliative care services had been involved at the end of life, whether the patient had made a request for euthanasia that was not granted and whether the patient had intentionally ended life without a physician's involvement by stopping 
eating and drinking, taking medication or otherwise. Demographic and clinical patient data were obtained from the death certificate data and linked anonymously after data collection.

\section{Analysis}

The response sample was first corrected for disproportionate stratification (by weighting each stratum to make the proportion in the response sample identical to the proportion in all deaths) and adjusted to be representative of all deaths in the first half of 2013 in terms of age, sex, marital status, province of death, cause of death and place of death (adjustments were needed for province of death and place of death). After this weighting procedure there were no significant differences between response sample and all deaths on any of these variables. Final weights varied between 0.11 and 1.90 . The same procedure was used in all survey years (in 1998 there was no disproportionate stratification). Weighted percentages, 95\% confidence intervals and $\mathrm{Chi}^{2}$ (two-sided) p-values were calculated with the complex samples function in SPSS 22.0.

\section{Supplementary results}

In cases resulting in euthanasia the request had been made both orally and in writing significantly more often in 2013 than in 2007 (62.8\% vs. 43.1\%, p<0.001) (Table S1). Another physician was consulted significantly more often in 2013 than in 2007 (92.6\% vs. 83.2\%, p=0.007). The decision was discussed with the family in $80.6 \%$ (95\% Cl 74.6-85.5) in 2013 compared with $77.4 \%$ in 2007. Neuromuscular relaxants and/or barbiturates were used to perform euthanasia in $64.8 \%$ in 2013 and in 52.1\% in 2007, with benzodiazepines and/or opioids used in $32.6 \%$ and $46.2 \%$ respectively ( $p=0.137)$. No changes in estimated life-shortening were found. Palliative care services had been involved in $73.7 \%$ of euthanasia cases in 2013 .

\begin{tabular}{|c|c|c|c|}
\hline Unweighted no. of cases & $\begin{array}{c}2007 \\
(n=142)\end{array}$ & $\begin{array}{c}2013 \\
(n=349)\end{array}$ & $\mathrm{Chi}^{2} \mathrm{p}$-value \\
\hline Explicit request from patient & 100 & 100 & - \\
\hline Form of the request & & & $<.001$ \\
\hline Only oral & $50.1(39.1-61.0)$ & $30.5(24.3-37.5)$ & \\
\hline Only in writing & $6.4(2.0-18.1)$ & $1.2(0.6-2.4)$ & \\
\hline Oral and in writing & $43.1(34.6-52.0)$ & $62.8(55.8-69.3)$ & \\
\hline Advance euthanasia directive & $0.5(0.1-3.4)$ & $5.5(3.0-9.7)$ & \\
\hline Consultation with other physician & $83.2(70.9-91.0)$ & $92.6(88.3-95.3)$ & .007 \\
\hline Discussion with family & $77.4(65.9-85.8)$ & $80.6(74.6-85.5)$ & .599 \\
\hline Drugs used for euthanasia/assisted suicide & & & .137 \\
\hline Neuromuscular relaxant and/or barbiturate(s) & $52.1(40.9-63.1)$ & $64.8(57.8-71.3)$ & \\
\hline Benzodiazepine(s) and/or opioid(s) & $46.2(35.3-57.5)$ & $32.6(26.3-39.6)$ & \\
\hline Other & $1.7(0.5-5.2)$ & $2.6(0.9-6.9)$ & \\
\hline Estimated degree of life shortening ${ }^{\dagger}$ & & & .934 \\
\hline Probably none & $1.7(0.5-5.2)$ & $1.7(0.7-4.1)$ & \\
\hline Less than $24 \mathrm{~h}$ & $9.7(5.9-15.6)$ & $12.5(8.5-18.1)$ & \\
\hline Less than 1 week & $44.1(33.3-55.4)$ & $41.0(34.4-48.0)$ & \\
\hline More than 1 week & 44.5 (33.7-55.9) & $44.8(38.1-51.6)$ & \\
\hline Palliative care services involved at end of life & $\ddagger$ & $73.7(67.5-79.1)$ & - \\
\hline
\end{tabular}

\footnotetext{
* Weighted column percentages (95\% confidence intervals).

† Estimated by the reporting physician.

¥ Not asked in 2007.
} 


\section{Study limitations}

Although our study uses a robust population-based sampling method, a number of study limitations have to be taken into account. While a fairly high response rate for physician surveys was achieved, we cannot exclude non-response bias. Yet analysis of non-response questionnaires revealed lack of time as the most frequent reason for non-participation. Recall bias may also have influenced results, even though physicians received the questionnaire no later than eight weeks after their patient's death. The sensitivity of the survey topic may have introduced untruthful or socially desirable reporting, but this is likely to be insignificant given the explicit guarantee of anonymity and physicians being well-acquainted with the survey.

\section{Competing interests statement}

The authors declare no competing interests. 